\title{
Surface Roughness Scattering of GaN Nanowire
}

\author{
Yang Peng \\ School of Science, Beijing Jiaotong University \\ BJTU \\ Beijing100044, China \\ e-mail: 12121656@bjtu.edu.cn
}

\author{
Lu Yan-Wu \\ School of Science, Beijing Jiaotong University \\ BJTU \\ Beijing100044, China \\ e-mail: ywlu@bjtu.edu.cn
}

\begin{abstract}
Electron mobilities limited by surface roughness scattering (SRS) in GaN nanowire have been investigated theoretically. The mobility found to be sensitive to the nanowire radius and electron density. Results show that the larger radius nanowires exhibit considerably higher mobility than smaller radius. Moreover, mobility limited by SRS found to be increase with increasing electron density. Results in this study can be used to design parameters of nanowire-based transisitors to obtain higher electron mobility.

Keywords: III-V compound semiconductor, mobility, surface roughness scattering, nanowire, GaN
\end{abstract}

\section{INTRODUCTION}

Semiconductor nanowires (SNWs) provide two-dimensional confinement to electrons in an dimension restricted in a few nanometers, and their high crystallinity makes them promising structures for photonic and electronic applications [1]. These reduced dimensional structures have been considered as alternative structures for future electronic devices [2,3,4]. Among these SNWs, III-V compound semiconductor (GaN, AlN, GaAs et. al) nanowires have attracted more attention due excellent properties of III-V compound semiconductors, and being proposed for a wide range of applications [4]. And the III-V compound semiconductor nanowire-based transistors are regarded as a promising route to continue the Moor's law of transistor scaling [1].

Scattering mechanisms, such as dislocation, interface roughness and surface roughness, which limit the mobility of electrons have been studied broadly in heterostructure quantum wells $[5,6,7,8]$. Among these scattering mechanisms, the surface roughness scattering (SRS) is proved to be the main limitation when the barrier is too thin $[7,8]$. Unlike the heterostructrue quantum wells structures, electrons in the nanowire-based transistors are surrounded by the surface, thus the SRS has a big influence on the electrons' mobility, especially when the nanowire has a small diameter. Therefore, the investigation on the SRS in nanowire structures is very important. However, there has not been sufficient investigations on the SRS in III-V nanowires. Jing Wang et. al have studied the SRS in the silicon nanowire transistors used a full three-dimensional quantum transport simulator [2], however, they have not investigated the influence of SRS on the electrons' mobility, which is one of the most important parameters in transistors. In this letter, we have investigated the mobility of electrons in nanowire limited by SRS. We assume that the surface roughness parameters in planar III-V compound materials, such as average roughness height and lateral size, are also suitable in nanowire structures. Even though our calculation is based on the GaN nanowire, the method used in this letter can be used in other semiconductor nanowire, such as Si, Ge, and GaAs.

\section{THEORETICAL MODELS AND METHODS}

In nanowires, the electrons are confined in the transverse direction, while they are free in the normal direction (z-direction). Thus, in cylinder coordinate system, the electrons' wave function is separable as $\varphi(r, \theta$, $z)=\varphi(r, \theta) e^{i k z}$, where $\varphi(r, \theta)$ is the solution of two-dimensional Shrodinger equation, which is:

$$
H \varphi(r, \theta)=\left[-\frac{\hbar^{2}}{2 m^{*}}\left(\frac{1}{r} \frac{\partial}{\partial r} r \frac{\partial}{\partial r}+\frac{1}{r^{2}} \frac{\partial^{2}}{\partial \theta^{2}}\right)+V(r)\right] \varphi(r, \theta)=E \varphi(r, \theta),
$$

where $\hbar$ is the reduced Plank constant, $m^{*}$ is the effective electron mass, $V(r)$ is the potential of the quantum well. Due to the circular symmetry, the wave functions should not have a dependence on the angle $\theta$, then the equation can be expressed as

$$
\left[-\frac{\hbar^{2}}{2 m^{*}} \frac{d^{2}}{d r^{2}}+\left(m^{2}-\frac{1}{4}\right) \frac{\hbar^{2}}{2 m^{*} r^{2}}+V(r)\right] R(r)=E R(r),
$$

where $R(r)=\varphi_{r}(r) r^{1 / 2}$. For a individual GaN nanowire, the channel can be treated as a infinite deep quantum well.

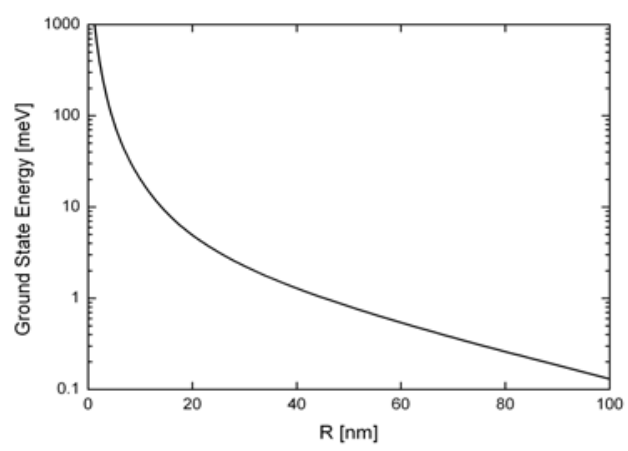

Figure. 1: Ground state energy level of GaN nanowire versus radius. 
Because the surface roughness can lead to fluctuations of the radius of nanowire, so in order to investigate the surface roughness scattering, it is necessary to know the dependence of the subband energy level on the nanowire radius. Fig. 1 shows the dependence of the ground subband energy level on the nanowire radius of $\mathrm{GaN}$. Result indicate that the ground subband energy level of GaN nanowire decrease as the value of nanowire radius $R$. If the influence of the surface roughness being taken into account, the nanowire radius $R$ can be written as: $R=R_{0}+\Delta(\theta, z)$, where the $\Delta(\theta, z)$ is the surface roughness with a Gaussian autocorrelation function [7,9 ]

$$
\langle\Delta(\theta, z) \Delta(\theta, z)\rangle=\Delta^{2} e^{-\frac{(R \theta)^{2}+z^{2}}{\Lambda^{2}}}=\Delta^{2} e^{-\frac{(R \theta)^{2}}{\Lambda^{2}}} e^{-\frac{z^{2}}{\Lambda^{2}}},
$$

where $\Delta$ is average roughness height, and $\Lambda$ is the roughness lateral size. The ground subband energy level fluctuation caused by the surface roughness can be written as

$$
\delta E_{0}=\frac{d E_{0}}{d R} \Delta(\theta, z) .
$$

Thus, the square of matrix element of the surface roughness scattering from $k$ state to $k^{\prime}$ state is

$$
|M|^{2}=\frac{1}{L_{z}}\left(\pi \Delta^{2} \Lambda^{2} e^{-q^{2} \Lambda^{2} / 4}\right)\left(\frac{d E_{0}}{d R}\right)^{2}\left(\int_{0}^{2 \pi} e^{-\frac{(R \theta)^{2}}{2 \Lambda^{2}}} d \theta\right)^{2},
$$

where $L_{z}$ is the length of nanowire. $q$ is one-dimensional wave vector as in one-dimensional scattering, which can be expressed as a function of $k$ and $k^{\prime}$. For simplicity, we assumed all the scatterings are elastic, which means the wave vector $/ k^{\prime} /=|k|=k_{f}=$ $\pi \times n$, in which $k_{f}$ is Fermi ware vector and $n$ is three dimensional electron density. Thus, the one-dimensional wave vector $q$ can be written as: $q=2 k \times \sin (\eta / 2)$, in which $\eta$ is angle between wave vector $k$ and $k^{\prime}$. In scattering process of one-dimension system, $\eta$ is $\pi$, which means $q$ is $2 k$.

The formula of scattering rate of the surface roughness scattering is

$$
\frac{1}{\tau(E)}=\frac{L_{z}}{2 \pi}\left(\frac{2 \pi}{\hbar}\right) \times \int_{\eta, k^{\prime}} M^{2} S_{C}(1-\cos \eta) \delta\left(E_{k^{\prime}}-E_{k}\right) d^{2} k^{\prime},
$$

where

$$
S_{C}=\left[\frac{|\ln (q \times 2 R)|^{-1}}{|\ln (q \times 2 R)|^{-1}+q_{s}}\right]^{2},
$$

is the screen factor and $q_{s}$ is the Thomas-Fermi wave vector written as $q_{s}=\frac{2 m e^{2}}{\varepsilon_{0} \varepsilon \hbar^{2}}\left(2 \pi^{2} n \times R\right)^{-1}$.
The mobility can be written as $\mu=\frac{e}{m} \tau$.

\section{RESULTS AND DISCUSSION}

Because the special structure characteristics of nanowire lead to some special properties of nanowire-based devices, it is necessary to investigate the structure dependent properties of these devices, and radius is one of the most important parameters in nanowire. The dependence of electrons mobility in GaN nanowire limited by SRS on nanowire radius is shown in Fig. 2, which indicates that the electron mobility increase with the increasing of nanowire radius. In this letter, we assume roughness height is $0.3 \mathrm{~nm}$ and roughness lateral size is $3 \mathrm{~nm}$ as fixed value. Roughness is remarkable in smaller radius case, which means stronger scattering hence lower electron mobility.

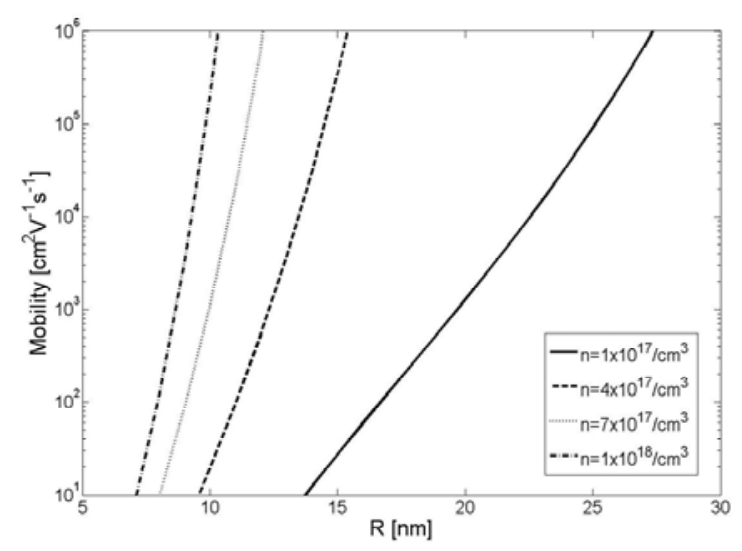

Figure. 2: Mobility limited by the SRS versus nanowire radius in $\mathrm{GaN}$ nanowire.

The result of Fig.2 is consistent with the experimental result given by Ref. 10: larger diameter nanowires exhibit significantly higher mobility than nanowires with smaller diameters.

Fig. 3 shows the result of mobility limited by SRS versus electron density. The value of three dimensional electron density of GaN nanowire is usually in the range $10^{17}-10^{18} \mathrm{~cm}^{-3}$ [11]. Mobility goes up with electron density increasing, for the reason that higher electron density indicate stronger screen effect, which is same as GaN heterojunction [7]. 


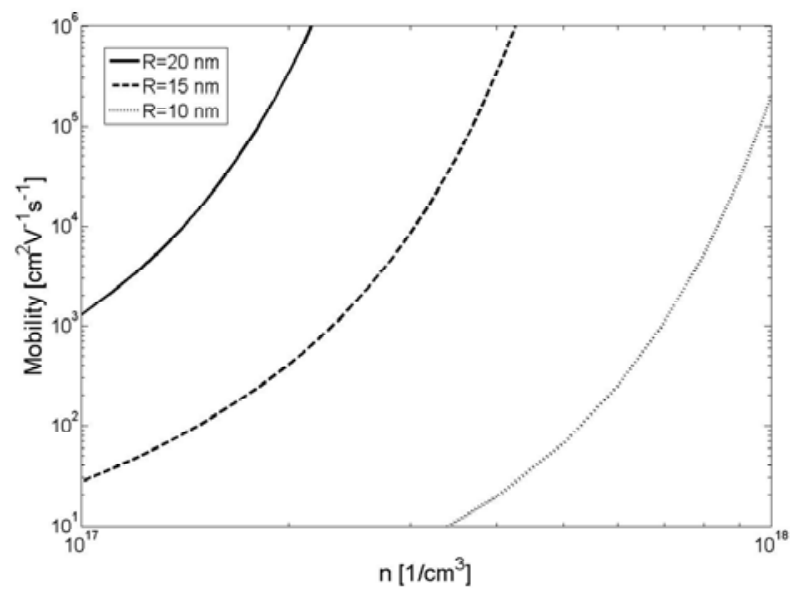

Figure. 3: Mobility limited by the SRS versus electron density in GaN nanowire.

\section{CONCLUSION}

In summary, we have studied the mobility limited by SRS in semiconductor nanowire. The distribution of electrons in individual GaN nanowire has been calculated by solving the Schrondger equation in a infinite deep quantum well. The ground energy level in GaN nanowires have been shown as a function of nanowire radius. The dependence of electron mobility limited by SRS have been found to be increase with the increasing and with increasing electron density, respectively. Results in this letter can be used to design parameters of semiconductor nanowire-based transistors to obtain higher electron mobility.

\section{ACKNOWLEDGEMENT}

Project supported by the National Natural Science Foundation of China (Grant Nos. 60976070 ) .

\section{REFERENCES}

[1] J. W. Yu, C. K. Li, C. Y. Chen, Y. R. Wu, L. J. Chou, and L. H. Peng, Appl. Phys. Lett., 99, 152108 (2011)

[2] J. Wang, E. Polizzi, A. Ghosh, S. Datta, and M. Lundstrom, Appl. Phys. Lett., 87, 043101 (2005)

[3] Y. Cui, Z. Zhong, D. Wang, W. U. Wang, and C. M. Lieber, Nano Lett., 3, 149 (2003)

[4] H. J. Joyce, Q. Gao, H. H. Tan, C. Jagadish, Y. Kim, J. Zou, L. M. Smith, H. E. Jackson, J. M. Yarrison-Rice, P. Parkinson, M. B. Johnston, Prog. Quantum Electron, 35, 23 (2011)

[5] D. Jena, A. C. Gossard, and U. K. Mishra, Appl. Phys. Lett., 76, 1707 (2000)

[6] B. Liu, Y. Lu, Y. Huang, G. Liu, Q. Zhu, and Z. Wang, Phys. Lett. A, 376, 1067 (2012)

[7] B. Liu, Y. W. Lu, G. R. Jin, Y. Zhao, X. L. Wang, Q. S. Zhu, and Z. G. Wang, Appl. Phys. Lett., 97, 262111 (2010)

[8] Y. Cao, H. Xing, and D. Jena, Appl. Phys. Lett., 97, 222116 (2010)

[9] R. K. Jana, and D. Jena, Appl. Phys. Lett., 99, 012104 (2011)

[10] A. Motayed, M. Vaudin, A. V. Davydov, J. Melngailis, M. He, and S. N. Mohammad, Appl. Phys. Lett., 90, 043104 (2007)

[11] C. Y. Chang, G. C. Chi, W. M. Wang, L. C. Chen, K. H. Chen, F. Ren, and S. J. Pearton, J Electronic Materials, 35, 738 (2006) 